\title{
Desgaste das facas do corte basal na qualidade da colheita mecanizada de cana-de-açúcar
}

\author{
Basecutter blades wear in quality of mechanized harvesting
}

\author{
Marcelo Tufaile Cassia ${ }^{I^{*}}$ Rouverson Pereira da Silva ${ }^{I I}$ Carla Segatto Strini Paixão ${ }^{I I I}$ \\ Rafael Scabello Bertonha ${ }^{\text {III }}$ Fábio Alexandre Cavichioli ${ }^{\mathrm{I}}$
}

RESUMO

Durante o processo de colheita mecanizada de canade-açúcar, o desgaste das facas do mecanismo de corte basal é fator diretamente relacionado com a qualidade do corte realizado pelas máquinas, sendo o emprego de ferramentas de controle de qualidade importantes no monitoramento deste processo. Assim, o presente trabalho, desenvolvido na região de Ribeirão Preto, teve por objetivo avaliar o desgaste das facas do corte basal $e$ os danos causados às soqueiras na colheita mecanizada de cana-de-açúcar, sob a ótica do controle estatístico de qualidade (CEQ). O desgaste das facas foi quantificado por meio da perda de massa e de suas dimensões, enquanto a qualidade do corte foi avaliada pela altura e pelos danos às soqueiras, classificados visualmente em função do nível de dano causado. Os resultados obtidos mostraram que o desgaste das facas ocorreu de maneira mais acentuada em determinados períodos de utilização, porém mantendo-se dentro de padrões de controle. A altura de corte não foi afetada pelo desgaste das facas, mantendo-se dentro dos limites de qualidade desejáveis para a operação. Finalmente, os danos causados às soqueiras variaram entre si em função de cada face de corte das facas avaliadas, havendo predominância de determinadas classes de danos em cada face de corte, porém sempre se mantendo dentro dos padrões aceitáveis pelo controle estatístico.

Palavras-chave: altura de corte, cartas de controle, controle estatístico de qualidade, danos às soqueiras.

\section{ABSTRACT}

During the sugarcane mechanized harvester process, the wear of base cutting knives is directly correlated to the quality of the cut made by the machines, and the use of quality control tools important in monitoring this process. Thus, the present study in Ribeirão Preto region aimed to assess the knives cut baseline and damage caused to wear brass knuckles in mechanized harvesting of cane raw sugar, from the viewpoint of statistical quality control (SQC). The wear of the knives was quantified by mass loss and its dimensions, while cutting quality was assessed by cutting height and damage to stumps visually classified according to the level of damage caused. The results showed that the wear of the knives was more pronounced in certain periods of use, but still within control standards. The cutting height was not affected by the wear of the knives, keeping within the limits of desirable quality for operation. Eventually damage the stumps ranged among themselves depending on each face of the cutting knives evaluated, and the predominance of certain classes of damage in each cutting face, but always remained in statistical control.

Key words: cut height, control charts, statistical quality control, ratoon damages.

\section{INTRODUÇÃO}

A adoção do sistema mecanizado na colheita de cana-de-açúcar já é o principal método de corte utilizado nas regiões produtoras e, principalmente, unanimidade nas áreas de expansão, não só por promover aumento da capacidade operacional como também adequação à legislação ambiental, por dispensar a queima prévia do canavial (PEREIRA \& TORROZAN, 2006). Na última safra colhida, a mecanização atingiu cerca de $70 \%$ da área de colheita no estado de São Paulo, sendo que, nas áreas de expansão, como nos estados de Goiás e Mato

\footnotetext{
IPrograma de Pós-graduação em Agronomia (Produção Vegetal), Faculdade de Ciências Agrárias e Veterinárias (FCAV), Universidade Estadual Paulista (UNESP), Via de Acesso Prof Paulo Donato Castellane, s/nº, 14884-900, Jaboticabal, SP, Brasil. E-mail: marcelocassia@gmail.com.

*Autor para correspondência.

IIDepartamento Engenharia Rural, FCAV, UNESP, Jaboticabal, SP, Brasil.

IIIPrograma de Pós-graduação em Agronomia (Ciência do Solo), FCAV, UNESP, Jaboticabal, SP, Brasil.
} 
Grosso, onde os canaviais se encontram em áreas planejadas, praticamente 100\% da área é colhida mecanicamente (ÚNICA, 2013).

De acordo com MELLO \& HARRIS (2003), as colhedoras realizam o corte basal da cana por impacto, usando um disco rotativo com múltiplas lâminas (facas), auxiliado por um rolo defletor que empurra a cana para frente antes de cortá-la e facilita a alimentação pelos rolos alimentadores. A deflexão e o corte basal são os principais responsáveis por danos na cana colhida e na soqueira da cultura, além de estar diretamente ligados à qualidade da matéria-prima e aos níveis de perdas na colheita, bem como prejudica a rebrota e podem causar redução na longevidade (SALVI et al., 2007; GRAY et al., 2009).

O controle de qualidade em operações agrícolas é destinado a detectar variações ou oscilações indesejáveis durante a execução de determinada operação, com base em indicadores pré-selecionados ou confrontando-os a padrões especificados, com a finalidade de inibir a ocorrência de falhas, evitando gastos desnecessários com ações corretivas (MILAN \& FERNANDES, 2002; SILVA et al., 2013). Trabalhos realizados por diversos autores, a exemplo de NEVES et al. (2006), CAMPOS et al. (2008), SILVA et al. (2008), SCHOGOR et al. (2009), e NORONHA et al. (2011), já enumeraram indicadores de qualidade para a operação de colheita mecanizada na cana-de-açúcar, concluindo que o monitoramento destes pode aumentar os níveis de qualidade da operação, mantendo a operação dentro de padrões desejáveis (NEVES et al., 2004; PELOIA et al., 2010).

Dessa forma, partindo da hipótese de que as faces das facas do corte basal possam influenciar os danos causados às soqueiras pela operação de colheita mecanizada e que o controle de qualidade possa manter estes danos em índices desejáveis, o presente trabalho teve por objetivo avaliar o desgaste das facas do corte basal e os danos causados às soqueiras na colheita mecanizada de cana-de-açúcar, sob o controle estatístico de qualidade (CEQ).

\section{MATERIAL E MÉTODOS}

O trabalho foi realizado no município de Guariba - SP, região de Ribeirão Preto, durante a colheita da safra 2008/09, em uma área comercial de cana-de-açúcar, próxima às coordenadas geodésicas de $21^{\circ} 25^{\prime} \mathrm{S}$ e $48^{\circ} 07^{\prime} \mathrm{W}$, altitude média de $530 \mathrm{~m}$, declividade média de $6 \%$ e clima Cwa, de acordo com a classificação de Köeppen. Foram avaliados dois talhões colhidos simultaneamente, nos quais se encontravam as variedades SP803280 e RB867515, em $4^{\mathrm{o}}$ e $5^{\mathrm{p}}$ corte, respectivamente.
Utilizou-se uma colhedora da marca Case-IH, modelo A7700, fabricada em 2002, equipada com motor de $184 \mathrm{~kW}$ (250cv) de potência nominal, rodados de esteiras com bitola de $1,88 \mathrm{~m}$ e operou durante o experimento à velocidade média de $6,4 \mathrm{~km} \mathrm{~h}^{-1}$. O mecanismo de corte basal dessa máquina é composto por dois discos que apresentam em suas extremidades cinco facas, sendo, neste trabalho, utilizadas facas retangulares de dois gumes de aço SAE 9260, com bordas lisas e dimensões iniciais de $220 \mathrm{~mm}$ de comprimento, espessura de 6,30mm, e massa média de $835 \mathrm{~g}$.

O porte do canavial foi verificado como caracterização da cultura antes da colheita, em 20 pontos para cada talhão, utilizando-se a metodologia proposta por RIPOLI (1996), classificando os colmos em eretos (ângulo com relação ao solo superior a $45^{\circ}$ ), acamados (ângulo entre 22,5 e 45) e deitados (ângulo abaixo de $\left.22,5^{\circ}\right)$. Para as demais avaliações, foi estabelecida uma malha amostral, composta por 72 pontos espaçados $50 \mathrm{~m}$ entre si, de área amostral delimitada por uma armação quadrada de metal, com 0,5m de lado.

A avaliação iniciou-se no momento seguinte à colocação das facas novas, sempre se avaliando a primeira e segunda linha de cana-deaçúcar colhida, eliminando possíveis influências do sentido de deslocamento da máquina. Determinou-se a altura de corte por meio da distância da superfície do solo ao ponto de corte em que a faca atingiu o colmo, conforme descrito por SILVA et al. (2008). Esta variável é importante para monitorar se a operação está ocorrendo de maneira adequada, sem que haja perda de matéria prima no campo, bem como arranquio de soqueiras pela ação da faca sob a superfície do solo.

O desgaste das facas de corte basal foi quantificado pela perda de massa (g) ao longo das inversões de faces de corte. As facas foram inicialmente identificadas e caracterizadas e, em seguida, separadas em dois grupos de cinco facas, que trabalharam sempre juntas em cada corte ao longo das avaliações.

Os danos causados à soqueira foram classificados de acordo com metodologia descrita por MELLO \& HARRIS (2003), sendo avaliados os colmos presentes dentro da armação anteriormente descrita em cada ponto amostral, os quais foram limpos e classificados visualmente por um único avaliador. Os danos causados pela operação foram considerados de três níveis: Sem Danos (SD), Danos Periféricos (DP) e Fragmentados (FR).

Os resultados foram analisados estatisticamente com o auxílio do programa Minitab $^{\circledR} 16$, passaram por uma análise exploratória 
(estatística descritiva) e análise de normalidade dos dados pelo teste Anderson-Darling, para verificar se os dados são bem modelados por uma distribuição normal ou se há a necessidade de transformação para sua normalização, bem como o comportamento da variabilidade. Realizou-se a análise de variância (ANOVA) fator único pelo teste F de Snedecor, com 5\% de significância, para verificar a existência ou não de diferenças significativas entre as médias das variáveis analisadas e, quando procedente, aplicou-se o teste de Tukey para comparação das médias.

Para avaliar a qualidade do processo, foram utilizadas cartas de controle para valores individuais (I-MR), que possuem limites de controle (inferior e superior) determinados em função da variabilidade do processo, que permitem inferir se há variação dos resultados devido a causas não controladas no processo (causas especiais) (MILAN \& FERNANDES, 2002).

\section{RESULTADOS E DISCUSSÃO}

Na tabela 1, são apresentados os parâmetros de medida de posição e tendência central (média e mediana) e de dispersão (amplitude, desvio padrão (DP) e coeficiente de variação (CV)) de estatística descritiva para a taxa de desgaste das facas, altura do corte basal e danos causados às soqueiras. Para a taxa de desgaste das facas, observa-se que a média e mediana encontraram-se próximas e, apesar do coeficiente de variação (CV) ser considerado médio a alto, os resultados apresentaram distribuição normal pelo teste de Anderson-Darling.

Para a altura de corte basal, os valores de média e mediana também se encontraram próximos.
Contudo, com valores de desvio padrão e CV(\%) muito elevados, indicando alta variabilidade entre os pontos avaliados, os valores apresentaram distribuição normal pelo teste de Anderson-Darling. Enquanto que os danos causados às soqueiras apresentaram valores de desvio padrão e CV muito elevados para todos os tipos, entretanto apenas para a parcela de soqueiras sem danos apresentaram grande variabilidade e, pelo teste de Anderson-Darling, os valores não se modelaram para uma distribuição normal, necessitando de transformação para a realização da análise de variância $(\log (x+1))$.

Na tabela 2, é apresentada a análise de variância para os índices avaliados, na qual a taxa de desgaste se mostrou mais acentuada nas faces de corte 1 e 4, o que pode estar relacionado com o maior período em que as facas de corte basal trabalharam em contato com o solo, devido às condições de preparo de solo do talhão ou do operador que trabalhou no período avaliado. De acordo com NEVES et al. (2004), o solo empolado pelas facas causa-lhe desgaste prematuro, danificando os rebolos, além de aumentarem os índices de impurezas no material colhido.

Para a altura de corte basal, a variável não foi influenciada pelas faces avaliadas, mostrando que o processo é controlado por outros componentes da colheita, contudo vale ressaltar que, em todas as situações, as médias de altura ficaram abaixo de $50 \mathrm{~mm}$, considerado como ideal para a operação. Segundo SALVI et al. (2007), para reduzir o índice de perdas de matéria-prima deixado no campo, o corte de base dos colmos não deverá ultrapassar a altura de $50 \mathrm{~mm}$, porém deve-se atentar para problemas nas soqueiras e contaminação por impurezas minerais.

Tabela 1 - Estatística descritiva para as variáveis taxa de desgaste das facas, altura do corte de base e danos causados às soqueiras: sem danos, danos periféricos e fragmentados.

\begin{tabular}{|c|c|c|c|c|c|}
\hline \multirow{2}{*}{ Parâmetros } & \multirow{2}{*}{ Taxa desgaste $\left(\mathrm{g} \mathrm{h}^{-1}\right)$} & \multirow{2}{*}{ Altura de corte (mm) } & \multicolumn{3}{|c|}{ - Danos às soqueiras (\%) -------- } \\
\hline & & & Sem Danos & Danos Perif. & Fragmentados \\
\hline Média & 3,51 & 37,78 & 20,97 & 45,07 & 33,96 \\
\hline Mediana & 3,49 & 35,38 & 18,33 & 42,26 & 33,33 \\
\hline Amplitude & 3,56 & 113,83 & 100,0 & 100,0 & 100,0 \\
\hline Desvio Padrão & 0,74 & 20,75 & 21,10 & 24,20 & 24,01 \\
\hline CV(\%) & 20,95 & 54,94 & 100,64 & 53,68 & 70,70 \\
\hline Cs(\%) & 0,19 & 1,19 & 1,01 & 0,48 & 0,31 \\
\hline $\mathrm{Ck}(\%)$ & 0,58 & 2,49 & 1,26 & $-0,08$ & $-0,41$ \\
\hline $\mathrm{AD}^{*}$ & $\mathrm{~N}$ & $\mathrm{~N}$ & - & $\mathrm{N}$ & $\mathrm{N}$ \\
\hline
\end{tabular}

CV: coeficiente de variação; Cs: coeficiente de assimetria; Ck: coeficiente de curtose.

*AD: teste de normalidade de Anderson-Darling (N: distribuição normal) 
Tabela 2 - Análise de variância e teste de médias para taxa de desgaste das facas, altura do corte de base e danos causados às soqueiras.

\begin{tabular}{llll}
\hline Fatores & $\begin{array}{c}\text { Taxa de } \\
\text { desgaste } \\
\left(\mathrm{g} \mathrm{h}^{-1}\right)\end{array}$ & $\begin{array}{c}\text { Altura de } \\
\text { corte }(\mathrm{mm})\end{array}$ & $\begin{array}{c}\text { Danos às } \\
\text { soqueiras } \\
(\%)\end{array}$ \\
\hline Face de corte (F) & $3,96 \mathrm{~b}$ & 33,4 & - \\
Face 1 & $2,84 \mathrm{a}$ & 40,6 & - \\
Face 2 & $3,19 \mathrm{a}$ & 42,7 & - \\
Face 3 & $4,04 \mathrm{~b}$ & 34,5 & - \\
Face 4 & & - & $20,97 \mathrm{a}$ \\
Tipo de dano (D) & - & - & 45,07 c \\
Sem danos & - & - & $33,96 \mathrm{~b}$ \\
Danos periféricos & - & & - \\
Fragmentados & & $0,466^{\mathrm{ns}}$ & - \\
Teste F (P>0,05) & & - & $0,001^{*}$ \\
F & $0,001^{*}$ & - & $0,042^{*}$ \\
D & - & 54,94 & 45,48 \\
F x D & - & &
\end{tabular}

${ }^{\text {ns }}$ Não significativo; ${ }^{*}$ Significativo pelo teste de F $(\mathrm{P}>0,05)$. Para cada fator, médias seguidas de mesma letra não diferem entre si, pelo teste de Tukey $(\mathrm{P}>0,05)$.

Para os danos causados às soqueiras, observou-se a predominância de danos periféricos seguidos por soqueiras fragmentadas, ficando os colmos sem danos em menor proporção em ralação aos demais, cenário oposto ao desejável (SILVA et al., 2008). Entretanto, os tipos de danos se comportaram de maneira diferenciada entre as faces de corte, havendo interação entre os fatores que estão desdobrados na tabela 3 .

Observa-se que não houve predominância de alguma face sobre a outra dentro de cada tipo de

Tabela 3 - Interação entre os fatores tipos de danos causados às soqueiras e faces das facas de corte basal.

\begin{tabular}{lrcr}
\hline & & & \\
Faces de corte & Sem danos & $\begin{array}{c}\text { Danos } \\
\text { periféricos }\end{array}$ & Fragmentados \\
& & $39,04 \mathrm{a}$ & $34,95 \mathrm{a}$ \\
Face 1 & $26,01 \mathrm{a}$ & $54,13 \mathrm{a}$ & $25,62 \mathrm{~b}$ \\
Face 2 & $20,25 \mathrm{~b}$ & $50,95 \mathrm{a}$ & $35,52 \mathrm{a}$ \\
Face 3 & $13,52 \mathrm{~b}$ & $36,16 \mathrm{a}$ & $39,76 \mathrm{a}$ \\
Face 4 & $24,07 \mathrm{a}$ & & \\
\hline
\end{tabular}

Médias seguidas de mesma letra na linha não diferem entre si pelo teste de Tukey $(\mathrm{P}>0,05)$. dano (Tabela 3), enquanto que, em cada face de corte, observou-se uma maior porcentagem de soqueiras com danos periféricos sobre os demais tipos de danos durante a colheita com a face 2 , fato que pode estar relacionado às condições da cultura durante este período (NORONHA et al., 2011). Para a na face 3, tanto os danos periféricos como os fragmentados foram superiores à parcela de soqueiras sem danos, que seriam os desejáveis para a operação (MELLO \& HARRIS, 2003).

Na figura 1a, são apresentadas as cartas de controle para a taxa de desgaste das facas do corte basal, quando o comportamento se manteve estável pelo controle estatístico, pois não houve ocorrência de pontos fora dos limites, superior e inferior de controle (LSC e LIC). Assim, compreende-se que, apesar de haver maior variabilidade entre as facas em algumas faces (face 2 e 4), o desgaste delas segue um padrão dentro de limites de controle, que está diretamente ligado ao controle de qualidade da produção destas para que se desgastem ao mesmo tempo, uma vez que trabalham em conjunto (GRAY et al., 2009).

Na figura 1b, são apresentadas as cartas de controle para a altura do corte de base, em que os resultados se mostraram estáveis pelo controle estatístico. Nota-se que, para todas as faces de corte, os resultados se comportaram de maneira semelhante, apresentando apenas na face 4 uma menor variabilidade dos dados, mas em todos os casos mostrando que o processo mantém padrão de qualidade (SILVA et al., 2013).

As cartas de controle para a porcentagem de danos causados às soqueiras, apresentadas na figura 2, mostram que, para todas as faces de corte e todos os tipos de danos, ocorre elevada variabilidade nos dados encontrados, ficando os limites estipulados normalmente no limite máximo especificado (LSE), porém mantendo os resultados obtidos dentro dos limites de controle, o que atribui estabilidade ao processo, independente da alta variabilidade (CAMPOS et al., 2008).

Quanto às faces de corte, observa-se assim como a taxa de desgaste apresentada na tabela 2. Durante a operação com as faces 2 e 3, predominaram os danos parciais sobre os demais (Figura 2b e 2c), enquanto, nas faces 1 e 4, os colmos fragmentados se igualaram aos danos parciais (Figura 2a e 2d). Para 


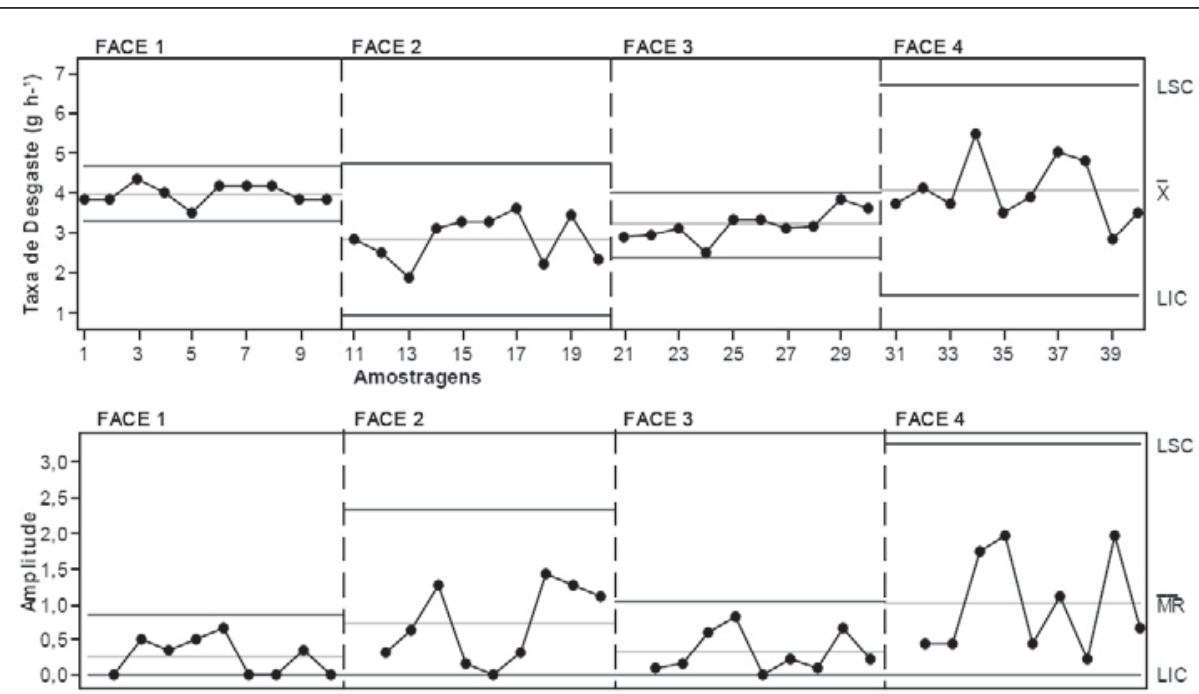

(a)
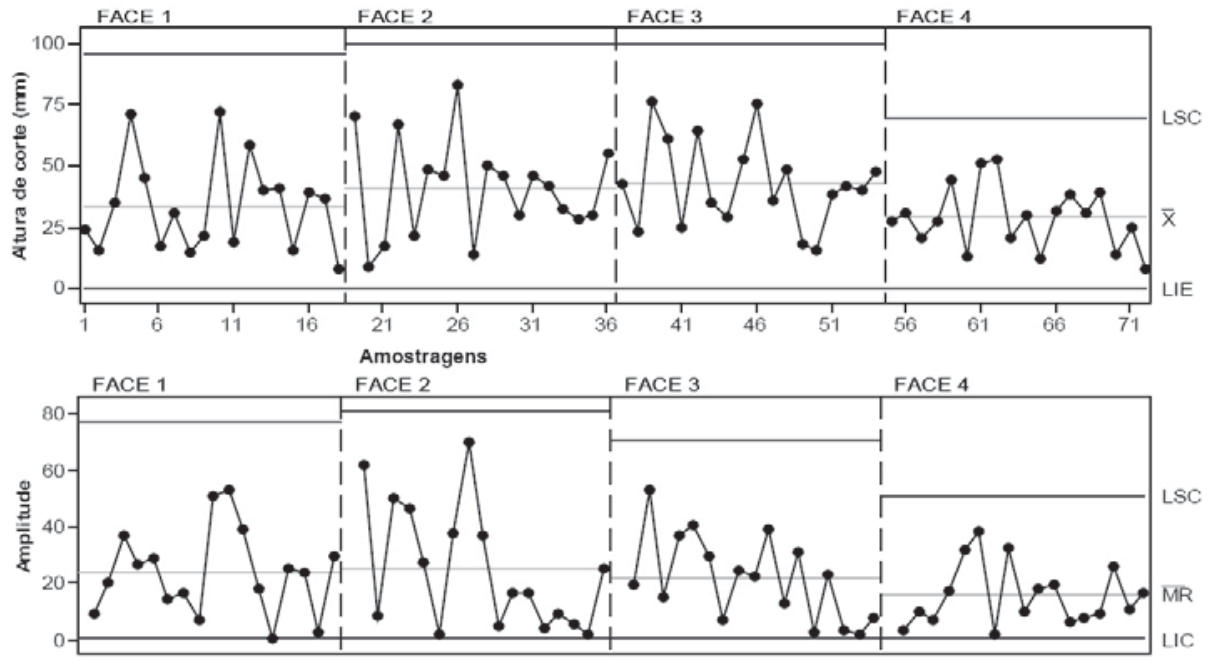

(b)

LSC: limite superior de controle

LIC: limite inferior de controle $\mathrm{X}$ e MR: linhas médias

Figura 1 - Cartas de controle para a taxa de desgaste das facas (a) e altura do corte basal (b), operando em cada face de corte na colheita mecanizada de cana-de-açúcar.

todas as faces de corte, as porcentagens de colmos sem danos se apresentaram sempre inferiores aos demais, fato que é indesejável para a operação de colheita mecanizada de cana-de-açúcar (MELLO \& HARRIS, 2003; SALVI et al., 2007; CAMPOS et al., 2008; GRAY et al., 2009; NORONHA et al., 2011).

\section{CONCLUSÃO}

O desgaste das facas do corte basal se mostrou mais acentuado em determinados períodos de períodos de uso, porém se mantendo sob controle durante toda sua utilização.

A altura de corte não variou ao longo do desgaste das facas, ficando dentro dos padrões desejáveis e dos limites estabelecidos pelo controle estatístico de qualidade para a operação.

Os danos causados às soqueiras variaram entre si em função de cada face de corte das facas avaliadas, havendo predominância de determinadas classes de danos em cada face de corte, porém sempre se mantendo dentro dos limites estabelecidos pelo controle estatístico. 


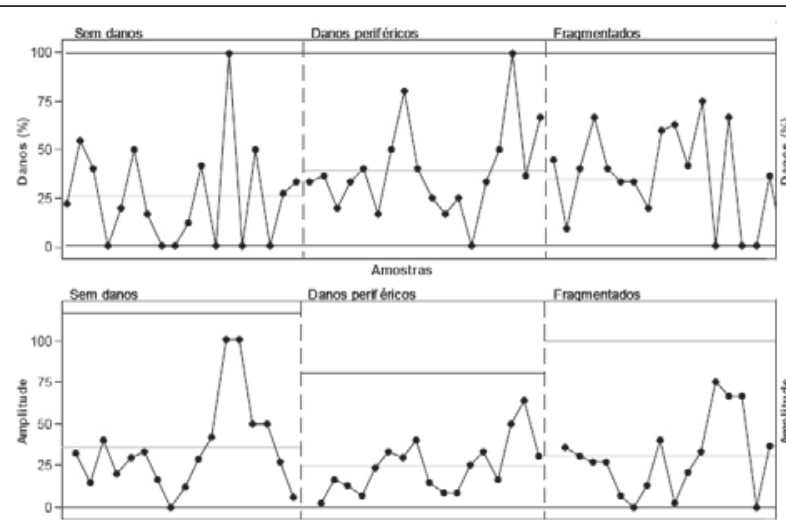

(a)

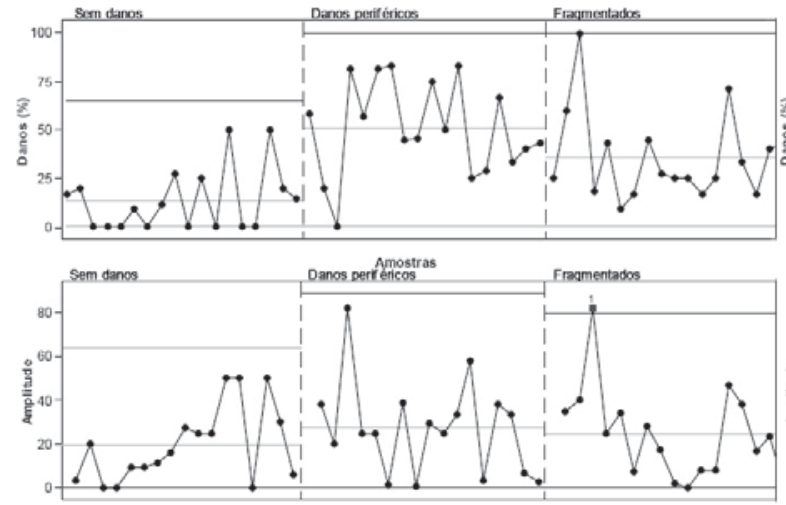

(c)

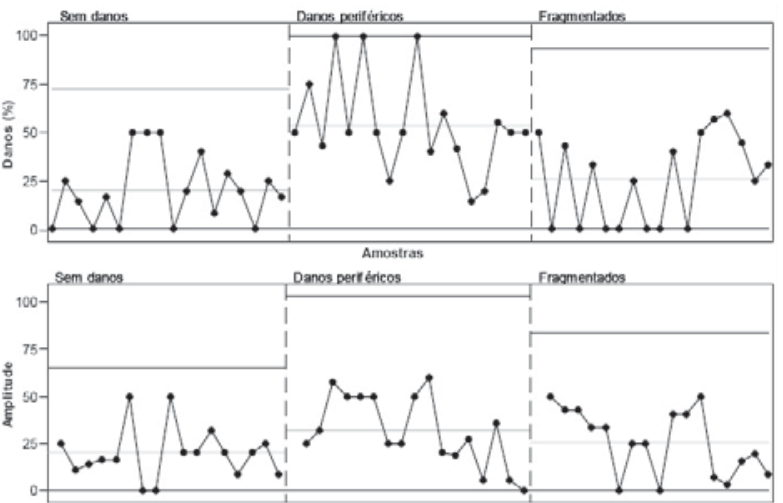

(b)

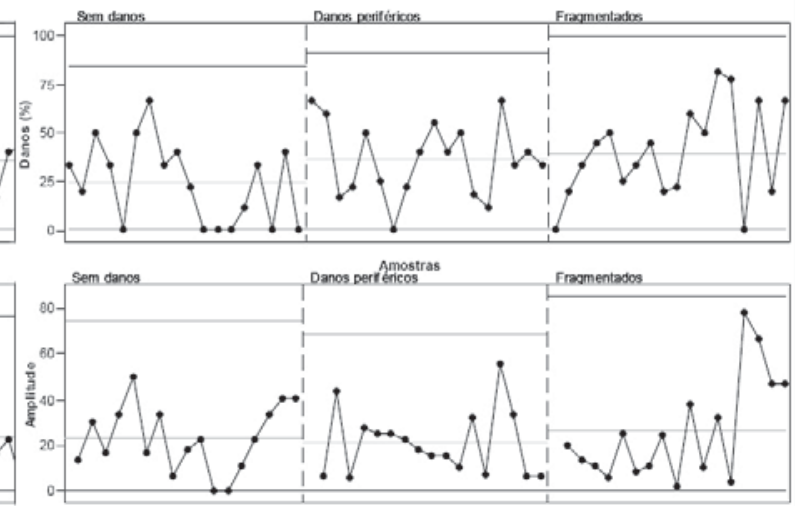

(d)

LSC: limite superior de controle LIC: limite inferior de controle X e MR: linhas médias

Figura 2 - Cartas de controle para a porcentagem de danos às soqueiras classificados em sem danos, danos periféricos e fragmentados, pelas faces das facas do corte basal: face 1 (a), face 2 (b), face 3 (c) e face 4 (d).

\section{REFERÊNCIAS}

CAMPOS, C.M. et al. Identificação e avaliação de variáveis críticas no processo de produção da cana-de-açúcar. Engenharia Agrícola, Jaboticabal, v.28, n.3, p.554-564, 2008. Disponível em: $<$ http://www.scielo.br/pdf/eagri/v28n3/a16v28n3.pdf>. Acesso em: 20 ago. 2013. doi: 10.1590/S0100-69162008000300016.

GRAY, G.R. et al. Suspensão pantográfica para corte de base de cana-de-açúcar. Ciência Rural, Santa Maria, v.39, n.3, p.766-771, 2009. Disponível em: <http://www.scielo. br/pdf/cr/v39n3/a96cr780.pdf>. Acesso em: 20 ago. 2013. doi: 10.1590/S0103-84782008005000096.

MELLO, R.C.; HARRIS, H. Desempenho de cortadores de base para colhedoras de cana-de-açúcar com lâminas serrilhadas e inclinadas. Revista Brasileira de Engenharia Agrícola eAmbiental, Campina Grande, v.7, n.2, p.355-358, 2003. Disponível em: <http://www. scielo.br/pdf/rbeaa/v7n2/v7n2a29.pdf>. Acesso em: 20 ago. 2013. doi: 10.1590/S1415-43662003000200029.

MILAN, M.; FERNANDES, R.A.T. Qualidade das operações de preparo do solo por controle estatístico de processo. Scientia Agricola, Piracicaba, v.59, n.2, p.261-266, 2002. Disponível em: <http://www.scielo.br/pdf/sa/v59n2/8919.pdf>. Acesso em: 20 ago. 2013. doi: 10.1590/S0103-90162002000200009.
NEVES, J.L.M. et al. Sistema de monitoramento de perdas visíveis de cana-de-açúcar em colhedora de cana picada. Engenharia Agrícola, Jaboticabal, v.24, n.3, p.764-770, 2004. Disponível em: <http://www.scielo.br/pdf/eagri/v24n3/a30v24n3.pdf>. Acesso em: 20 ago. 2013. doi: 10.1590/S0100-69162004000300030.

NEVES, J.L.M. et al. Avaliação de perdas invisíveis na colheita mecanizada em dois fluxos de massa de cana-de-açúcar. Engenharia Agrícola, Jaboticabal, v.26, n.3, p.787-794, 2006. Disponível em: $<$ http://www.scielo.br/pdf/eagri/v26n3/16.pdf>. Acesso em: 20 ago. 2013. doi: 10.1590/S0100-69162006000300016.

NORONHA, R.H.F. et al. Controle estatístico aplicado ao processo de colheita mecanizada diurna e noturna de cana-de-açúcar. Bragantia, Campinas, v.70, n.4, p.931-938, 2011. Disponível em: <http://www.scielo.br/pdf/brag/v70n4/28.pdf>. Acesso em: 20 ago. 2013. doi: 10.1590/S0006-87052011000400028.

PELOIA, P.R. et al. Capacity of the mechanical harvesting process of sugar cane billets. Scientia Agricola, Piracicaba, v.67, n.6, p.619-623, 2010. Disponível em: <http://www.scielo. br/pdf/sa/v67n6/v67n6a01.pdf>. Acesso em: 20 ago. 2013. doi: 10.1590/S0103-90162010000600001. 
PEREIRA, L.L.; TORREZAN, H.F. Colheita mecanizada da canade-açúcar. In: SEGATO, S.V.etal.(Org.). Atualizaçãoemprodução de cana-de-açúcar. Piracicaba: Livroceres, 2006. p.333-346.

RIPOLI, T.C.C. Ensaio \& certificação de máquinas para colheita de cana-de-açúcar. In: MIALHE, L.G. Máquinas agrícolas: ensaios \& certificação. Piracicaba: Fundação de Estudos "Luiz de Queiroz”, 1996. Cap.13, p.635-673.

SALVI, J.V. et al. Avaliação do desempenho de dispositivo de cortes de base de colhedora de cana-de-açúcar. Engenharia Agrícola, Jaboticabal, v.27, n.1, p.201-209, 2007. Disponível em: $<$ http://www.scielo.br/pdf/eagri/v27n1/14.pdf>. Acesso em: 20 ago. 2013. doi: 10.1590/S0100-69162007000100014.

SCHOGOR, A.L.B. et al. Perdas das frações de canade-açúcar submetida a diversos métodos de colheita. Revista Brasileira de Zootecnia, Viçosa, v.38, n.8, p.1443-1450, 2009. Disponível em: <http://www.scielo. br/pdf/rbz/v38n8/07.pdf>. Acesso em: 20 ago. 2013. doi: 10.1590/S1516-35982009000800007.

SILVA, R.P. etal.Controle estatístico aplicado aoprocesso decolheita mecanizada de cana-de-açúcar. Engenharia Agrícola, Jaboticabal, v.28, n.2, p.292-304, 2008. Disponível em: <http://www.scielo. br/pdf/eagri/v28n2/a10v28n2.pdf>. Acesso em: 20 ago. 2013. doi: 10.1590/S0100-69162008000200010.

SILVA, R.P. et al. Qualidade da colheita mecanizada de feijão (Phaseolus vulgaris) em dois sistemas de preparo do solo. Revista Ciência Agronômica, Fortaleza, v.44, n.1, p.61-69, 2013. Disponível em: <http://www.scielo.br/pdf/rca/v44n1/ a08v44n1.pdf>. Acesso em: 20 ago. 2013. doi: 10.1590/S180666902013000100008

ÚNICA (UNIÃO DA INDÚSTRIA DE CANA-DE-AÇÚCAR). Indústria Brasileira de Cana-de-açúcar: uma trajetória de evolução. Acesso em: 15 ago. 2013. Online. Disponível em: <http://www.unica.com.br/linhadotempo/index.html>. 


\section{ERRATA}

Artigo publicado com a grafia do autor Rafael Scabello Bertonha incorreta 